\title{
Managing depression in primary health care centers: The highlights of the program and further improvements
}

\author{
Herlina Siwi Widiana ${ }^{1}$, Lenore Manderson ${ }^{2}$, Katrina Simpson ${ }^{3}$ \\ ${ }^{1}$ Faculty of Psychology, Universitas Ahmad Dahlan, Indonesia \\ ${ }^{1,3}$ School of Psychological Sciences, Monash University, Australia \\ ${ }^{2}$ School of Public Health, University of Witswaterand, South Africa \\ ${ }^{1}$ herlina.widiana@psy.uad.ac.id, , ${ }^{2}$ lenore.manderson@wits.ac.za, ${ }^{3}$ simpsonkate2@gmail.com
}

\section{ARTICLE INFO}

Article history

Received 3 November 2019

Revised 24 February 2020

Accepted 26 February 2020

\section{Keywords}

clinical psychologist

depression

management

primary health care

\begin{abstract}
Many people with probable depression present at primary health care centers (PHC) with physical complaints. Clinical psychologists (CPs) in Sleman district and Yogyakarta city, Special Province of Yogyakarta, Indonesia, have been integrated into PHC to provide psychological services. The study on which we draw aimed to explore how depression is managed in PHC. Thirty four CPs and twenty people diagnosed with mild to moderate depression participated in semi-structured interviews. Based on the thematic analysis of their transcribed interviews, seven themes emerged: community education and support, referral pathways, assessing depression, diagnosis, communicating a diagnosis, accepting diagnosis, and treatment of depression. The co-location of CPs with other medical services at the PHC level supports community understandings of mental health problems. It also encourages community members to use PHC services and to have access to various behavioral interventions for people with depression. Recommendations and directions for further research are discussed.
\end{abstract}

\section{Introduction}

Globally, depression is the third leading cause of disease burden and is projected to be the first by 2030 (Ferrari et al., 2013; Lopez \& Mathers, 2006; WHO, 2011). More than 10\% of people with depression worldwide present at primary health care centers (PHC) (DosSantos, Huang, Menezes, \& Scazufca, 2016; Milanovic, Erjavec, Poljicanin, Vrabec, \& Brecic, 2015; Udedi, 2014). Although depression commonly manifests in psychological symptoms such as fluctuations in emotions and cognitions (Alang, 2016; Hitchcock et al., 2016; Molina et al., 2014), people with depression often report physical complaints (Brintnell, Sommer, Kuncoro, Setiawan, \& Bailey, 2013; Kleinman, 2004; Seifsafari, Firoozabadi, Ghanizadeh, \& Salehi, 2013; Selim, 2010). However, if only the physical complaints are treated, and the psychological issues are not addressed, depressive symptoms may continue. The integration of psychological services in PHC not only provides access but also acknowledges the value of these services (Hepworth et al., 2015; Knowles, Chew-Graham, Adeyemi, Coupe, \& Coventry, 2015; Sadock, Auerbach, Rybarczyk, \& Aggarwal, 2014).

Co-location, as one type of integrated care, has been implemented to manage depression in primary care practices with GPs, nurses and psychologists in North West England, where each professional has his or her own role according to their expertise (Knowles et al., 2015). In this program, patients meet with the GP and nurse for medical services and with the psychological well-being practitioner for their emotional or mental health condition. Other than co-location, collaborative care has proven effective in managing depression in the US (Gilbody, Bower, Fletcher, Richards, \& Sutton, 2006) by enhancing the capacity of primary 
care physician providers, through involving a depression case manager with a mental health background and a psychiatrist to provide feedback to the depression case manager (Katon \& Unutzer, 2006).

In Indonesia, 6\% of Indonesians aged 15 years old and older - around 15 million people - is reported to experience emotional disorders (Kementerian Kesehatan, 2013). This figure primarily refers to depression and anxiety symptoms, derived from household sample responses to a Self-Reporting Questionnaire (SRQ) included in the Basic Health Research Survey of the Ministry of Health of Indonesia, a national government department which organizes health affairs. Probably it is an underestimate, but even if not, it suggests that a significant number of people need assistance through PHC.

The Ministry of Health of Indonesia previously advised PHC (known as Puskesmas) to respond to mental health needs by referring patients to mental hospitals for treatment, but it now emphasizes on treating patients within the community (Marchira, 2011), or in PHC settings, either as an inpatient or as an outpatient, rather than in psychiatric hospitals. As a result of changes in the mental health program, the role of the PHC has broadened to include the provision of mental health services. Rather than only referring people with apparent mental health problems to a general hospital or a psychiatric hospital, PHC now provides a psychological assessment, diagnosis and treatment for community members. Therefore clinical psychologists (CPs) have been integrated into the PHC system as local government staff in some districts in Yogyakarta, Jakarta and Surabaya, as a result of local government initiatives. The current study was conducted in Sleman district and Yogyakarta city in the Special Region of Yogyakarta; the three other districts in this region, CPs had not yet been integrated in the PHC system, when the research was conducted. At this time, one CP is posted in each PHC in the aforementioned two districts.

Previous studies have been conducted to explore the integration of CPs in the PHC. The integration of CPs in the PHC centres in Sleman District was beneficial in terms of improving mental health among community member (Isfandari et al., 2012; Sari, 2016). The CPs work not only with high-risk patients and patients with psychological problems but also with healthy patients through outreach and home-visits in the community, and in outpatient consultations at the clinic (Setiyawati, Blashki, Wraith, Colucci, \& Minas, 2014). However, there is no study on managing depression in the PHC in the lens of CPs and people with depression. Therefore this study explores the perspectives of CPs on how depression is managed in these centers, as well as the perceptions and accounts of experiences of those with depression. Below, we describe the integration of psychological services in PHC settings, especially in managing depression, and offer recommendations for further implementation programs.

\section{Method}

Qualitative methods was used in this study. In-depth interviews were primarily chosen to explore how depression is managed in PHCs, by gathering information on the perceptions and experiences of people with depression and the CPs who work in the PHC centers.

\section{Participants}

The participants of the study on which this article is based consisted of two groups: clinical psychologists and people with depression.

Clinical psychologist $(C P)$. Request letters of participation and explanatory statements were provided to the clinical psychologists who were advised that they could contact the first author by email, text or phone, if they were interested in participating. Thirty four CPs working in PHCs in Sleman district and Yogyakarta city (21 and 13 centers, respectively), of whom only two were male, were recruited to participate in the study. Those working in Sleman district 
had a longer mean length of time working than those in Yogyakarta city (4 years vs. 1.5 years). The CPs gave written consent to their participation.

People with depression. The first author identified and recruited a clinical sample with recommendations from the CPs. Twenty Javanese adults, who have been diagnosed with mild to moderate depression by a CP in a PHC from less than one year to more than four years ago participated in this study (mean since time of diagnosis: 39 months); all but one were Muslim, aged $18-55$ years, with majority female (16 out of 20). Participants diagnosed with mild to moderate depression gave verbal consent, reflecting their reluctance to complete and sign the written consent forms primarily for data security.

\section{Procedures}

The first author conducted some extended semi-structured interviews with the CPs on the management of depression in PHC centers, using an interview guide with some questions in identifying the features of people presenting to the centers for treatment. The interview guide evaluated the experiences of CPs on mental health, including the assessment process, management, and treatment of depression in PHC centers in Sleman district and Yogyakarta city.

Following this, those Javanese adults were interviewed using the Cultural Formulation Interview (CFI) as an interview guide (APA, 2013) to gain an understanding of how depression is experienced from patients' perspectives. The CFI is a tool developed by the DSM-5 cultural issues subgroup to ensure the cultural validity of the DSM-5 (Aggarwal, 2013) to enable a better psychiatric diagnosis of people who have illnesses that incorporates cultural factors (Worcester, 2013).

Amendments in the current research around the CFI meant that, instead of a 16-item template, eight additional questions of the supplementary module 1 of CFI were included to align with Kleinman's (1980) idea of an explanatory model of illness. Components included in this explanatory model are the nature of illness, perceived cause, severity, stigma, expected course, impact, fears related to the disorder, help seeking and treatment sought, and desired outcomes (Johnson, Chin, Kajumba, Kizito, \& Bangirana, 2017). In addition, the first question was changed, from "What brings you here today?" to "What is your illness?" Where applicable, the word "problem" was replaced with "illness".

All interviews were audiotaped and then transcribed verbatim by the first author into Javanese, Indonesian or both, depending on the language chosen by participants for their interview. In this article, all quotes have been translated into English.

\section{Data Analysis}

Inductive thematic analysis (Braun \& Clarke, 2006) was applied in data analysis. Transcribed data from the interviews were read and reread by the first author to be engaged with the data. In the next step, notes were made based on the data. Following steps and considerations outlined by Bryman (2004), codes were generated manually for each interview by the first investigator. The next step was to reread the transcripts to generate codes, and to review the codes to ensure the description of the same phenomenon occurred across transcripts if there was a connection between codes and consideration of general linkages in the data.

A similar process was applied to each transcript although this was done separately. Initially, for the clinical sample and the CPs, a list of codes was gathered. Preliminary and final codes and code definitions were entered into separated codebooks with the examples of participants' statements and a review of any notes. At this point, all authors reviewed the codes and discussed the emergent themes to achieve consensus for each group.

The next step was to integrate the data from codes, and the emergent themes from both groups to determine consistency across the two groups. The themes developed and 
subthemes that characterized participants' experiences in relation to treatment seeking, diagnosis and management of depression were further evaluated. For data gathered from people with depression, only themes related to how depression was managed in the PHC centers are presented in this article.

\section{Results}

The results analysis of qualitative interviews suggested that managing depression in primary health centers, according to the study participants, starts with CPs educating community members about various mental health problems, including depression. The following sections examine the qualitative findings for the major themes and subthemes in greater detail. In presenting quotations from the interview texts, the responses of clinical psychologists have been identified by the abbreviation "CP" after each quote; the responses of people with depression have been identified by the abbreviation "PD" after each quote. Therefore, CP1 refers to clinical psychologist number $1, \mathrm{CP} 2$ refers to clinical psychologist number 2 and so on. Similarly, PD1 indicates person with depression number 1, PD2 indicates person with depression number 2 and so on.

\section{Community education and support}

Stigma. Both CPs and people living with depression explained that in the community, mental health problems known as "craziness" (Indonesia: gila; Javanese: edan), reflected by social withdrawal and other antisocial behavior, are managed through institutionalization and this is considered as a standardized and appropriate practice. As one participant explained: "People around me keep saying to me, you should be put in a psychiatric hospital" (PD8).

Participants spoke of concerns about disclosure when presenting for care. Thus, one participant spoke of his embarrassment when seeing a CP regularly at the PHC: "Why was I, a man, frequently in the clinic? Many people asked what my disease was. I was embarrassed when people knew I was coming here" (PD1).

Cultural understanding. The local understandings of the etiology and symptoms of depression are commonly linked to the action of bad spirits. These spirits are typically Javanese place-based, or are believed to be the outcome of ill wishes from someone else in the community. This led people to seek a help and an advice from traditional healers (Indonesian: dukun; orang pintar), religious leaders, and/or religious healers. Such local treatment at times occurs simultaneously with care provided by a psychiatrist at the psychiatric hospital, or from a CP at a PHC.

People with depression would meet religious leaders frequently to share their problems and seek advice. In addition to provide personal advice on managing mental health or interpersonal problems, religious leaders often suggest to those seeking their counsel that they undertake some good deeds, such as reciting the Qur'an and dhikr (repeated recitation of the name of Allah, also referred to asmaul husna). Religious healers, in contrast to religious leaders, also give treatment. One common treatment is ruqya in which a healer reads Qur'anic verses to negate the disruptive impact of bad spirits and manage the supernatural world, as well as the personal worlds of cognitive dissonance and existential crises. One participant explained her experience of being ruqya:

"Javanese people believe that [I] was being troubled by a bad spirit, as I was confused, remained in my room, and was afraid and cried all the time, for about five months. My uncle called a man who did ruqya twice on me. And then I got my spirit back and gradually I could think [clearly] again" (PD10). 
Religious coping. In line with advice from religious leaders and religious healers, fifteen of twenty participants diagnosed with depression explained that they managed their depression through religious coping. As most participants are Muslim, they reported that they prayed, remembered Allah (dhikr), recited and listened to the Qur'an, and tried to gain a greater understanding of the Qur'an. One participant explained what this involved:

"Prayer and long breath, but the prayer is more for relief. Prayer with long sujood [English: prostration]. It is impossible for Allah not to have noticed my prayers" (PD9).

Education by CPs. Because of people's unfamiliarity with mental health problems such as depression, when CPs were first integrated into the PHC, they worked hard to increase mental health awareness, to reduce stigma, and to encourage people to use their services. This outreach included community education programs at monthly meetings held in village halls. As one CP explained: "In the beginning of my career as a CP in this district, I tried to give psycho education to villagers, emphasizing that mental health problems need not be a severe psychiatric disorder. I would explain that depression could be mild, moderate or severe" (CP3). CPs also trained mental health cadre on mental health issues especially related to screening and enhancing the content of this training with early interventions such as stress management, relaxation, effective communication and counseling skills. The aim was for cadre to provide an appropriate support and mentoring on first contact with a person who might be depressed, before they decided to refer them to a CP at the PHC.

"I tried to strengthen cadre skills. As a result, cadre reported mental health problems in their village, and I was able to access the community through regular community social gatherings, youth meetings and meetings of the elderly. Gradually, people understood that they could meet with a CP at the PHC center for any problems that caused them distress. Initially, the number of visits to my clinic was low, so I needed to actively engage with community members to change their mindset. Now I am more passive, working in the clinic, as people come directly to me when they have mental health problems" (CP3).

Health promotion was also considered necessary to enhance the community understanding of depression and to strengthen family support for people with depression:

"For depression, [we] needed to educate the family to help people with depression, and there should be more health promotion programs" (CP7).

\section{Referral pathways}

People with depression meet CPs at the PHC in one of two referral pathways: from a clinician inside the PHC, or a clinician and/or mental health cadre from outside the PHC.

Referral from internal PHC. People with depression may be referred to a $\mathrm{CP}$ by internal paramedic or member of the medical staff, such as the GP, midwife, or dentist situated in the PHC center. Most people with depression who were referred to CPs had previously presented to a GP with physical complaints. Some GPs referred patients directly to CPs when they identified symptoms of depression. Others were referred only after receiving medication and meeting the GP on several occasions, when their physical complaints had still not resolved.

The explanation provided by the GP to the patient, before their referral to the $\mathrm{CP}$, played an important role in the patient's readiness for psychological care. Many patients rejected being referred to a $\mathrm{CP}$, and even those who accepted this tended to deny that they had any psychological problem and were reluctant to talk with the CP. In some PHCs, even at the time of the study, when CP presence was well established, some people remain 
reluctant to accept care from a $\mathrm{CP}$. One $\mathrm{CP}$ spoke of negative community perceptions of them:

"I got patients with depression mostly [referred] from the GP. When they were referred to me, sometimes they did not understand why. The patients tended to see people referred to a $\mathrm{CP}$ in the clinic as crazy, so numerous patients refused to talk to me, insisting: "I am not crazy" (CP2).

When the GP explained to a patient that he or she did not have a physical illness, but their problem was psychological, the patient tended to be more willing to talk with the CP:

"[The GP usually said] you do not have any [physical] illness, it is related to your mind. I have a friend, a CP upstairs, you can meet the CP. The CP will train you to do relaxation; you need to be relaxed. You also can talk to the CP. If you relax, have no tension, no stress, you will be healthy" (CP4).

As a standard procedure in the PHC center, pregnant women in the last three months of pregnancy, who meet midwives for regular check-ups, should also be referred to the CP to ensure their readiness for childbirth and the arrival of an infant. One participant, who was depressed during her pregnancy, explained: "I was in the third trimester of my pregnancy; the midwife referred me to the $C P$. The $C P$ was very welcoming. I told her the story behind my pregnancy [while unmarried, and her boyfriend refused to marry her]. I cried in front of her" (PD9).

Referral external to the PHC. In each village, mental health cadre plays a significant role in reporting, referring and supporting people with depression to see the CP. When a cadre finds a community member with clear anomalies in attitude and behavior, he or she will encourage the community member to go to the PHC center, and report to the $\mathrm{CP}$ by phone, text or in person; often cadre will accompany a person to the PHC. In cases when people with depression cannot come to the PHC center because of the severity of his or her condition, then, based on information from the cadre, the $\mathrm{CP}$ will conduct a home visit. In addition, GPs from outside the PHC, including private GPs or midwifes, also referred patients to CPs in the PHC. As one participant stated: "I went to see the GPs, traditional healers, religious leaders and finally the midwife. Then, I was advised by the midwife to meet the psychologist in the PHC' (PD12).

Self-referral. Increasingly over time, people with depression have come directly to the $\mathrm{CP}$ in the PHC centers, sometimes alone, other times with a family member. Patients who come directly to a CP without referral are categorized at the health center as APS (Indonesian: Atas Permintaan Sendiri, i.e. self-referral). Self-referrals have become increasingly common, as people have gained information from family, friends, and mental health cadres, and by searching on the internet about CP services in the PHCs. One participant in this study, for instance, was actively looking for information about the nearest $\mathrm{CP}$ service from the internet and found a $\mathrm{CP}$ at the local PHC.

Interaction CP with GP. When working with people with depression, CPs are required to collaborate with GPs. 7 of 34 CPs explained cooperative interaction with the GP, including with discussions about particular patient conditions. As a CP stated:

"The relationship [between me and the GP] is cooperative and mutual. I usually go down to the outpatient room to see the patients if they are unable to come here [upstairs at CP's room]" (CP1). 
Handover. Nine CP participants explained the handover process from GPs to the CPs, although this varied between PHCs. Some used a paper based procedure in which the GP wrote the referral and then the patient met the $\mathrm{CP}$ with the referral letter. An alternative handover procedure involved the GP escorting the patient to meet the CP. The GP asked the patient to wait outside the CP's room and had a conversation with $\mathrm{CP}$ before inviting the patient to come in, as one participant explained: "Usually, the GP communicates with me first. The GP comes with the patient and explains his/her medical record to me" (CP10).

Refer to psychiatrists. For severe depression or depression with suicidal ideation, the National Health Service protocol still requires referral to a psychiatrist at the district hospital or to the psychiatric hospital. This procedure is also necessary if a person with depression does not respond to treatment provided by a CP, or improvement with medication from a GP. In such cases, the patient may need additional medication and other interventions. CPs communicate with the GP, and the GP then refers the patient to the psychiatrist. Conversely, if the psychiatrist either from a district hospital or from the psychiatric hospital decides that a patient is improving and no longer needs psychiatric support, but require continuation of medication, this may be managed by the GP with CP support at the PHC. One participant explained: "At first, the patient got psychotropic medicine and the dosage was determined by the psychiatrist. After three months of seeing a psychiatrist, the patient was allowed to just meet the GP and CP in the PHC and got their medicine from the center" (CP17).

\section{Assessing Depression}

The use of clinical interviews and clinical observations. In assessing depression, all CPs conducted a clinical interview and clinical observation during an interview session with patients. One participant stated, "I mostly [use] clinical observation on the way he/she speaks, his/her gaze and affect" (CP11). As the CPs work in health settings in which most people come to seek help for their physical condition, they initiate the assessment by asking the patient about their physical symptoms. One participant explained: "First, I asked about their physical condition as described in the referral from downstairs [from the GP], and then explored with follow-up questions" (CP33).

The use of assessment tools. 23 of 34 CPs stated that they sometimes used an assessment tool when working with patients who they suspected had depression. Most CPs used the Beck Depression Inventory (BDI) when they suspected that the client might have depression, administering the BDI as a self-inventory, or using only certain items in the BDI as an interview guide. Two CPs stated that they used other inventories: the Hamilton Depression Rating Scale (HDRS) or Hopkins Symptom Checklist (HSC).

Barrier in using assessment tools. One reason why CPs rarely use psychological assessment tools is that they are unavailable for routine use in the PHCs; CPs who do use inventories, use their own. However, CPs have reported difficulties in using the BDI when, for instance, a patient may not understand certain items, and they have to explain the items to the patient. Some patients may not want to fill out the inventory. Another reason is the extra cost to the patient of using any inventory, including the BDI. This increasing cost of assessment may be beyond what the patient must pay, in contrast to the cost if assessment is based solely on clinical interview and clinical observation, when the patient would pay only the basic cost for CP services.

\section{Diagnosis}

Identify depression. The majority of CPs used the Pedoman Penggolongan Diagnosis Gangguan Jiwa III (PPDGJ III; Manual for the Classification and Diagnosis of Mental 
Disorders; Departemen Kesehatan, 1993), following the symptoms listed in the PPDGJ III to diagnose and categorize mild, moderate or severe depression. The PPDGJ III is the latest version of the manual, although it was issued in 1993. It is based on the ICD-10, with multiaxial diagnosis using DSM-IV. In the PPDGJ III, depression is included under the category of mood disorder. Depression, with code F32, has three variants, mild (F32.0), moderate (F32.1) and severe (F32.2). CPs diagnose patients with depression if three main symptoms of depression are found: chronic fatigue, flat affect, and loss of happiness.

Communication between $\boldsymbol{C P}$ and GP. Five CPs explained the communication process with GPs in making diagnoses. Some GPs would visit the CP, themselves, to explain the patient's condition and discuss the possibility that they might have depression. Following assessment and diagnosis, the $\mathrm{CP}$ would communicate the diagnosis to the GP. The $\mathrm{CP}$ would make the diagnosis if the assessment matched the criteria of depression, as one participant explained: "Sometimes we have a different diagnosis. No problem, as long as we communicate it. I speak to the GP and say that [the patient] has depression" (CP33).

There were variations in the referral diagnosis. Some GPs wrote depression as a firm diagnosis. Others might include a question mark around depression on the medical record. Sometimes, patient referrals from the GP had an alternative possible diagnosis, such as anxiety or a psychosomatic condition. For example:

"When the patient was referred, the initial diagnosis written in the medical record was F41 anxiety, or F45 psychosomatic. But if main symptoms of depression were found, I will meet the GP to discuss. Finally, it was diagnosed with F32 depression" (CP7).

Barriers in diagnosing. Some CPs and GPs tended to diagnose anxiety or an unspecified psychosomatic disorder rather than depression, except when a person presented with severe depression and suicidal ideation. They are cautious about diagnosing depression because treatment should follow strict guidelines. In addition, CPs had difficulties differentiating mild depression from other mental problems: "In my experience, [I diagnose] depression when it is severe depression. For mild [depression] I had difficulty in differentiating whether the person had a psychosomatic disorder or depression" (CP8).

This difficulty in diagnosing may be the result of the limited time that CPs have to work with their patients. Some patients only met a CP for one session, and did not want to return for further sessions. In such cases, the $\mathrm{CP}$ has to assess the patient and make a diagnosis based on a single session only, and they could not check and evaluate their diagnosis. Moreover, a CP only works with a patient for only around 30 minutes: "We have a limited time; an hour or two hours is too long [in the PHC] because the queues are long" (CP3).

\section{Communicating a diagnosis of depression}

Considering patients' characteristics. After a diagnosis, CPs are faced with challenges of how best to communicate it, and the relevance of that diagnosis to the patient. If the $\mathrm{CP}$ feels that the patient will be further depressed if they are told that they have depression, then he or she may choose to withhold the diagnosis. Mostly, CPs do not communicate the diagnosis directly, but rather communicate with the patient's family and educate the family to give support to the patient. As one participant explained:

"I consider the patient's condition, and I need to be careful how I communicate [the diagnosis of] depression. If I have to communicate it, [I prefer] to speak to his or her family. I worry that [if I tell them], the patient will feel an even greater burden and this will affect their healing" (CP16). 
Withheld diagnosis. Seven CPs explained that they tend to withhold the diagnosis even though the patient asked them directly: "If the patient is not aware and open, I will use another sentence [not depression] and explain the symptoms" (CP31).

However, most patients asked the $\mathrm{CP}$ for advice to solve their problems, emphasizing what they should do. Only a few asked specifically about their illness. As one participant said; "I did not ask [what my illness was]" (PD4).

\section{Accepting diagnosis of depression}

Denying. Most patients who were told they had depression found it difficult to accept the diagnosis because they saw depression as synonymous with being "crazy." As described above, considerable stigma still continues against all mental health problems in Indonesia, including depression, as one participant explained: "Depression is like you are crazy [gila]. Someone with depression looks like they have lost their brain. You lose your heart, lose your brain. It is like you've gone crazy, lost your mind" (PD11).

Fluctuating condition. In the process of accepting that they have depression, patients reported that they experienced fluctuations in their condition. They were questioning: "Why do I have this? Why do I have this illness?" (PD13). However, while at times they found the diagnosis concerning, they also found it helpful: "Sometimes I can accept it, I am grateful and accepting [Indonesian: ikhlas], maybe there is wisdom gained from this experience. People have different tests, some have a physical illness, and some have ill will. Eventually, I can accept it" (PD13).

\section{Treatment}

Evidence-based treatments. A broad range of treatments were given to people diagnosed with depression by CPs in the PHC centers, including psycho education, relaxation techniques, supportive therapy, cognitive therapy, behavior activation, and cognitive behavior therapy in the PHC centers. CPs considered the condition and the characteristics of the person when choosing a therapy. Psycho education was usually given to link physical symptoms reported by patients with their psychological condition, especially to those who had been referred to the CP by a GP or other paramedic. Many types of relaxation such as breathing, imagery and muscle relaxation were given to patients who reported physical symptoms during their depression. Supportive or cognitive therapy was provided in the first session as a brief intervention, especially if the CP felt that the patient would be unwilling to return for further sessions. When people with depression agreed to come for a follow-up session or attend a series of sessions, the CP might use behavior activation or cognitive behavior therapy, beginning with a contract with the patient that specified the number of treatment sessions and the willingness of the patient to follow the program.

Barrier in treating. People with depression who had insight into their health and were motivated to take action to recover from their depression tended to return for further treatment. However most people with depression, especially those who had been referred, were unwilling to receive continued therapy. They gave several reasons: they did not have time as they had to work, they had no-one to accompany them, they did not have transport, or they could not afford the cost of the CP services:

"Patients who present in this PHC are low income... They feel the cost of psychological service is expensive and so they do not think that coming again is important because the money can be used for something else" (CP27).

When people diagnosed with depression did not return for a second session with the $\mathrm{CP}$, the $\mathrm{CP}$ could not evaluate the effectiveness of the treatment already provided. If the 
patient returned, the $\mathrm{CP}$ would start the session by evaluating the patient and asking about their ability to follow the treatment, such as relaxation at home.

As the patient begins to improve, the $\mathrm{CP}$ involves the mental health cadre to ensure support in the community after the clinic program concludes. The mental health cadre then reports on the patient's progress to the $\mathrm{CP}$. On rare occasions, a person with depression may come on his or her own accord after they recover from their depression, if they believe they are having a relapse, and they take steps to meet with the $\mathrm{CP}$ again.

Religious therapy. In addition to treatment programs, some CPs also suggest therapy based on the religion of the patient, advising them to be grateful, and to remember Allah through dhikr and Salah. One participant explained her experience: "The CP said that I might not be grateful. I wanted a husband; [God] gave me one. I wanted a child; [God] gave me one. Have I been grateful? Oh... I might lack gratitude, and so I have been given gratitude therapy" (PD12). The CP asked the patient to write down things for which she should be grateful each day. Muslims believe that Allah will provide when a person is grateful, and so this therapeutic approach made sense in terms of local cultural values.

Suicidal management. When a person is experiencing depression and has had suicidal ideation, the standard procedure is that the $\mathrm{CP}$ communicates to the GP, who will then refer the patient to a psychiatrist at the district hospital or the psychiatric hospital. In some PHC centers with inpatient facilities, a CP in collaboration with the GP may offer a person with depression the option of hospitalization at the PHC. If a person has had suicidal ideation and attempted suicide in the past 24 hours, the CP educates the family about alertness to suicidal tendency and asks them to keep company with the person with depression. Medication is only used in cases of severe depression, and as prescribed by the treating psychiatrist.

CPs have different ways of approaching patients to gain information on suicidal ideation. Some ask family members whether the patient has had suicidal ideation or attempted suicide, because they are afraid to ask the patient directly, lest their question precipitate an attempt. Others, however, ask directly, as one participant explained,

"The GP and I should screen for suicide. [I] have to ask, so I know whether the patient had had suicidal ideation because he or she seems to have little hope. [I ask] What is your plan to commit suicide? [If he or she answers], it's just an idea, then we have to address this. We have to evaluate, is it urgent? Should he or she be hospitalized or referred [to a psychiatrist]? [The patient] is not allowed to go back home. [If he or she] wants to go home, he or she should fill in and sign a consent form" (CP4).

\section{Discussion}

The results of this study provide data on how depression is managed in the primary health care centers. Based on the results, the highlights of the program and areas for further improvement are discussed below.

\section{The highlights of the program}

Psychological services in the PHC centers at Sleman district and Yogyakarta city are colocated, with psychological and medical services situated in a single building. A referral system, mainly from GPs to CPs, stimulates communication between CPs and medical personnel. CP may communicate with the GP, or other medical staff, that refer patients with psychological problems to the $\mathrm{CP}$, either for additional information, or to arrange for the $\mathrm{CP}$ and other provider to see a patient together at the clinic or at a home visit. This approach reflects a growing understanding of mental health services in the clinics. Further research is 
needed to ascertain the most appropriate depression care that accommodates the local cultural context and health systems.

A cultural understanding is important in managing mental health problems, including depression. Research elsewhere has shown the need for culturally appropriate assessment and interventions in mental health (Illes, Grace, Nino, \& Ring, 2015). As described above, in assessing depression in PHCs, some CPs use the Beck Depression Inventory, although some found that not all items were appropriate for their patients. Data from study participants with depression in Indonesia (Widiana, Manderson, \& Simpson, 2018) have indicated depression manifests in both universal and unique ways through internalized and externalized emotions, loss of interest in social and religious activities, disturbance in cognition, the presence of physical symptoms, and suicidal ideation. This indicates the need for a culturally appropriate depression assessment tool for use in PHC settings, in line with suggestions from Brintnell and colleagues (2013). In selecting the assessment tools for evaluating depression in Indonesian patients, the measures must be adapted to ensure that they accurately capture expressions of depression specific to Indonesians patients. The Indonesian Depression Checklist (IDC) was developed and principally based on Javanese adult experiences of depression, and so does effectively accommodate culturally-specific aspects of depression (Widiana, Simpson, \& Manderson, 2018).

In treating depression at Indonesian PHCs, other than by giving therapies that are commonly used internationally, some CPs use a religious approach to depression, mainly based on Islam, such as remembering Allah and "gratefulness" therapies. Some studies have found connections between religiosity (belief and adherence to practices) with mental health status. Among people who are particularly religious, religious coping appears to reduce depression levels (AbdAleati, Zaharim, \& Mydin, 2016; Pargament, Tarakeshwar, Ellison, \& Wulff, 2001). Especially for Muslims, attending the mosque (for men), reading Al Qur'an and Hadith, prayer, fasting during Ramadhan, and Islamic based psychotherapy, all appear to reduce depression (Azhar \& Varma, 1995; Townsend, Kladder, Ayele, \& Mulligan, 2002). Drawing on these findings, in managing depression among Indonesians, CPs may find it helpful to apply therapy based on the patients' religious beliefs, because of the acceptance of this approach and the improvements evident for patients. However, in line with Meer and Mir (2014), CPs need to be trained to enhance their knowledge of religious beliefs and cultural issues, and whether to work inside or outside the clinic, including draw on religious perceptions in therapy.

Cadres have an important role in managing depression, as they bridge the community with the CPs in the PHCs. The beneficial effect of these cadres was demonstrated when the use of mental health cadres was implemented after the devastating tsunami in Aceh (see Boothby, Veatch, \& Pentes, 2011). In this study area, the mental health training given to cadres effectively enhanced their knowledge and understanding of mental health. Mental health cadres were able to identify the need for psychological services in affected communities and better able to refer people to CPs in the PHCs in that region. Mental health cadres give support to individuals in their villages, and report to PHC staff if they find a community member who they believe has signs or symptoms of mental health problems. As a result, the number of people who access psychological services has increased over time.

\section{Areas for further improvement}

In the Special Region of Yogyakarta, by far the largest ethnic group is Javanese (95.63\% of the total population; Ananta, Arifin, Hasbullah, Handayani, \& Pramono, 2015). Javanese traditional healing practices are based on Muslim views, ideas about personhood, and the causes of poor health. In this context, traditional healers (dukun) and religious leaders (ustad) are believed to have the ability to cure people from a range of ailments, including psychosomatic conditions and mental disorder (Woodward, 1985). As noted above, in local beliefs about illness etiology among Javanese and elsewhere in Indonesia, depression is 
believed to be caused by bad spirits (Boothby, Veatch, \& Pentes, 2011), whereby patients seek help from traditional or religious healers. CPs need to work with traditional healers and religious leaders as well as with mental health cadre to increase community understandings of mental health problems and to ensure the effectiveness of formal referral systems. Based on previous research in Indonesia, educating traditional healers and religious leaders appears important as a way to accelerate behavior change and to build local partnerships of care (Sarwono, 1996).

Challenging stigma is crucial to bridging access barriers in the community (Boothby, Veatch \& Pentes, 2011). Although health promotion programs conducted by CPs appear to be effective in reducing stigma in some PHCs, stigma still influences the willingness of patients to be referred to CPs in other PHCs. This indicates the need for more work to address stigma in the community, to increase knowledge or change attitudes and behavior related to mental health problems (Thornicroft et al., 2016). Previous research suggests that educational interventions were effective in reducing stigma in the community (Mehta et al., 2015), and in reducing stigma among health workers (Henderson et al., 2014). A previous study among primary health workers in Kenya shows too that the more knowledgeable they are on mental health, the more positive mental health worker attitudes toward individuals with mental health problems (Mutiso et al., 2017). CPs need to increase communication with GPs and other clinicians in the clinics to enhance their understanding of mental health and the CP's role, which in turn increases referrals.

Cost is another barrier that works against the use of psychological services, and this includes the additional cost for psychological assessment. CPs tend to avoid using assessment tools because patients have problems in paying for this. Therefore, CPs relied on the results of clinical interview and clinical observation. The IDC described above was developed as part of the management of depression in the PHC centers (Widiana, Simpson, \& Manderson, 2018). CPs may use the IDC as a guide to interviewing patients, thereby improving assessment, without additional cost to the patients.

CPs have different approaches in assessing suicidal intention. Some CPs directly asked the patients, while others preferred to ask the patient's family. However, CPs tend to avoid asking about suicidal tendency for fear that their question might trigger a suicide attempt, although the published literature demonstrates that directly asking about suicidal tendency does not increase the suicide intention, but rather reduces suicidal intentions and improved mental health outcomes (Dazzi, Gribble, Wessely, \& Fear, 2014). However, training on assessment of suicidal tendency is needed as part of assessment and management of mental health problems (Bajaj et al., 2008). Therefore, further research is necessary to explore the best way to assess suicide within the local Indonesian culture.

This study only involved CPs and people with depression. GPs, nurses and other medical staff were not involved; therefore, research that includes health professionals in addition to CPs may yield greater insight into health providers' perceptions and experiences of managing depression in PHC settings. Based on the current programs, the management of depression in PHC settings by CPs may extend to other districts in the Special Province of Yogyakarta and other PHC centers in Indonesia. As a first step in that direction, however, it will be necessary to integrate CPs into those PHC that do not yet have them.

\section{Conclusion}

The integration of CPs in PHC settings is a step forward in enhancing mental health services in the community and encouraging community understandings of mental health programs and their management. One further direction for better services is to foster cultural understandings of depression in assessing and treating people with depression by CPs and other medical staff, so that they will be able to enhance management of depression in local PHC and community settings. 


\section{References}

AbdAleati, N. S., Zaharim, N. M., \& Mydin, Y. O. (2016). Religiousness and mental health: Systematic review study. Journal of Religion \& Health, 55(6), 1929-1937. https://doi.org/10.1007/s10943-014-9896-1

Aggarwal, N. K. (2013). Cultural psychiatry, medical anthropology and the DSM-5 field trials. Medical Anthropology, 32, 393-398. https://doi.org/10.1080/01459740.2013.776047

Alang, S. M. (2016). "Black folk don't get no severe depression": Meanings and expressions of depression in a predominantly black urban neighborhood in Midwestern United States. Social Science \& Medicine, 157, 1-8. https://doi.org/10.1016/j.socscimed.2016.03.032

Ananta, A., Arifin, E. N., Hasbullah, M. S., Handayani, N. B., \& Pramono, A. (2015). Demography of Indonesia's ethnicity. Singapore: Institute of Southeast Asian Studies.

APA. (2013). American Psychiatric Association: Diagnostic and Statistical Manual on Mental Disorder (5th ed.). Arlington, VA: American Psychiatric Association.

Azhar, M. Z., \& Varma, S. L. (1995). Religious psychotherapy in depressive patients. Psychotherapy and Psychosomatics, 63(3-4), 165-168. https://doi.org/10.1159/000288954

Bajaj, P., Borreani, E., Ghosh, P., Methuen, C., Patel, M., \& Crawford, M. J. (2008). Screening for suicidal thoughts in primary care: The views of the patients and the general practitioners. Mental Health in Family Medicine, 5, 229-235.

Boothby, N., Veatch, M., \& Pentes, M. (2011). Evaluating of treatment of Axis I mental health disorders in Aceh, Indonesia. The Psychiatric Online, 35, 248-255. https://doi.org/10.1192/pb.bp.110.030205

Braun, V., \& Clarke, V. (2006). Using thematic analysis in psychology. Qualitative Research in Psychology, 3(2), 77-101. https://doi.org/10.1191/1478088706qp063oa

Brintnell, E. S., Sommer, R. W., Kuncoro, B., Setiawan, G. P., \& Bailey, P. (2013). The expression of depression among Javanese patients with major depressive disorder: A concept mapping study. Transcultural Psychiatry, 50(4), 579-598. https://doi.org/10.1177/1363461513501709

Bryman, A. (2004). Social research methods (2nd ed.). New York: Oxford University Press.

Dazzi, T., Gribble, R., Wessely, S., \& Fear, N. T. (2014). Does asking about suicide and related behaviours induce suicidal ideation? What is the evidence? Psychological Medicine, 44(16), 331-336. https://doi.org/10.1017/S0033291714001299

Departemen Kesehatan. (1993). Pedoman Penggolongan dan Diagnosis Gangguan Jiwa di Indonesia III (Manual for the Classification and Diagnosis of Mental Disorders in Indonesia III). Jakarta: Direktorat Jenderal Pelayanan Medik Departemen Kesehatan Republik Indonesia.

DosSantos, E. R., Huang, H., Menezes, P. R., \& Scazufca, M. (2016). Prevalence of depression and depression care for populations registered in primary care in two remote cities in the Brazilian Amazon. Plos One, 11(3), 1-13. https://doi.org/10.1371/journal.pone.0150046

Ferrari, A. J., Charlson, F. J., Norman, R. E., Patten, S. B., Freedman, G., Murray, C. J. L., $\&$ Whiteford, H. A. (2013). Burden of Depressive Disorders by Country, Sex, Age, and Year: Findings from the Global Burden of Disease Study 2010. Plos Medicine, 10(11). https://doi.org/10.1371/journal.pmed.1001547

Gilbody, S., Bower, P., Fletcher, J., Richards, D., \& Sutton, A. J. (2006). Collaborative care for depression - A cumulative meta-analysis and review of longer-term outcomes. 
Archives of Internal Medicine, 166(21), 2314-2321. https://doi.org/10.1001/archinte.166.21.2314

Henderson, C., Noblett, J., Parke, H., Clement, S., Caffrey, A., Gale-Grant, O., . . ., \& Thornicroft, G. (2014). Mental health-related stigma in health care and mental healthcare settings. Lancet Psychiatry, 1(6), 467-482. https://doi.org/10.1016/S22150366(14)00023-6

Hepworth, J., Askew, D., Foley, W., Duthie, D., Shuter, P., Combo, M., \& Clements, L. A. (2015). How an urban Aboriginal and Torres Strait Islander primary health care service improved access to mental health care. International Journal for Equity in Health, 14, 51-58. https://doi.org/10.1186/s12939-015-0183-x

Hitchcock, C., Mueller, V., Hammond, E., Rees, C., Werner-Seidler, A., \& Dalgleish, T. (2016). The effects of autobiographical memory flexibility (memflex) training: An uncontrolled trial in individuals in remission from depression. Journal of Behavior Therapy and Experimental Psychiatry, 52, 92-98. https://doi.org/10.1016/j.jbtep.2016.03.012

Illes, R. A. C., Grace, A. J., Nino, J. R., \& Ring, J. M. (2015). Culturally responsive integrated health care: Key issues for medical education. International Journal of Psychiatry in Medicine, 50(1), 92-103. https://doi.org/10.1177/0091217415592368

Isfandari, S., Rahmawati, T., Siahaan, S., Roosihermiatie, B., Abbas, I., Afiatin, T., \& Maslim, R. (2012). Evaluasi penempatan psikolog dalam pelayanan kesehatan jiwa di Puskesmas Kabupaten Sleman, Yogyakarta, Indonesia, 2011 (Evaluation of the presence of psychologist for mental health service at Primary Health Cares in Sleman District, Yogyakarta, Indonesia,. Buletin Penelitian Sistem Kesehatan, 15(4), 354-359.

Johnson, L. R., Chin, E. G., Kajumba, M., Kizito, S., \& Bangirana, P. (2017). Views on depression from traditional healing and psychiatry clinics in Uganda: Perspectives from patients and their providers. Journal of Cross-Cultural Psychology, 48(2), 243-261. https://doi.org/10.1177/0022022116675424

Katon, W., \& Unutzer, J. (2006). Collaborative care models for depression - Time to move from evidence to practice. Archives of Internal Medicine, 166(21), 2304-2306. https://doi.org/10.1001/archinte.166.21.2304

Kementerian Kesehatan. (2013). Riset Dasar Kesehatan 2013 (Basic Health Research 2013). Jakarta: Badan Penelitian dan Pengembangan Kementerian Kesehatan Republik Indonesia.

Kleinman, A. (1980). Patients and healers in the context of culture: An exploration on the borderland between Anthropology, Medicine and Psychiatry. Berkeley: University of California Press.

Kleinman, A. (2004). Culture and depression. The New England Journal of Medicine, 351(10), 951-952. https://doi.org/10.1056/NEJMp048078

Knowles, S. E., Chew-Graham, C., Adeyemi, I., Coupe, N., \& Coventry, P. A. (2015). Managing depression in people with multimorbidity: a qualitative evaluation of an integrated collaborative care model. BMC Family Practice, 16, 32-41. https://doi.org/10.1186/s12875-015-0246-5

Lopez, A. D., \& Mathers, C. D. (2006). Measuring the global burden of disease and epidemiological transitions: 2002-2030. Annals of Tropical Medicine and Parasitology, 100(5-6), 481-499. https://doi.org/10.1179/136485906X97417

Marchira, C. R. (2011). Integrasi kesehatan jiwa pada pelayanan primer di Indonesia: Sebuah tantangan masa sekarang. (Integration mental health into community health care in Indonesia: A review of current challenges). Manajemen Pelayanan Kesehatan, 14(3), $120-126$. 
Meer, S., \& Mir, G. (2014). Muslim and depression: The role of religious belief in therapy. Journal of Integrative Psychology and Therapeutics, 2(2), 1-8. https://doi.org/10.7243/2054-4723-2-2

Mehta, N., Clement, S., Marcus, E., Stona, A. C., Bezborodovs, N., Evans-Lacko, S., \& Thornicroft, G. (2015). Evidence for effective interventions to reduce mental healthrelated stigma and discrimination in the medium and long term: systematic review. British Journal of Psychiatry, 207(5), 377-384. https://doi.org/10.1192/bjp.bp.114.151944

Milanovic, S. M., Erjavec, K., Poljicanin, T., Vrabec, B., \& Brecic, P. (2015). Prevalence of depression symptoms and associated socio-demographic factors in primary health care patients. Psychiatria Danubina, 27(1), 31-37.

Molina, M. A. L., Jansen, K., Drews, C., Pinheiro, R., Silva, R., \& Souza, L. (2014). Major depressive disorder symptoms in male and female young adults. Psychology Health \& Medicine, 19(2), 136-145. https://doi.org/10.1080/13548506.2013.793369

Mutiso, V. N., Musyimi, C. W., Nayak, S. S., Musau, A. M., Rebello, T., Nandoya, E., \& Ndetei, D. M. (2017). Stigma-related mental health knowledge and attitudes among primary health workers and community health volunteers in rural Kenya. Journal of Social Psychiatry, 63(6), 508-517. https://doi.org/10.1177/0020764017716953

Pargament, K. I., Tarakeshwar, N., Ellison, C. G., \& Wulff, K. M. (2001). Religious coping among the religious: The relationships between religious coping and well-being in a national sample of Presbyterian clergy, elders, and members. Journal for the Scientific Study of Religion, 40(3), 497-513. https://doi.org/10.1111/0021-8294.00073

Sadock, E., Auerbach, S. M., Rybarczyk, B., \& Aggarwal, A. (2014). Evaluation of integrated psychological services in a university-based primary care clinic. Journal of Clinical Psychology in Medical Settings, 21(1), 19-32. https://doi.org/10.1007/s10880013-9378-8

Sari, E. P. (2016). Upaya pemberdayaan komunitas melalui sinergi kader posyandu dan psikolog puskesmas (Community empowerment through synergy between posyandu cadres and psychologists in the PHC). Jurnal Ilmiah Psikologi Terapan, 4(1), 53-61.

Sarwono, S. (1996). Personalistic belief in health: A case in West Java. In P. Boomgaard, R. Sciortino, \& I. Smith (Eds.), Health care in Java: Past and Present (pp. 81-91). Leiden: KITLV Press.

Seifsafari, S., Firoozabadi, A., Ghanizadeh, A., \& Salehi, A. (2013). A symptom profile analysis of depression in a sample of Iranian patients. Iranian Journal of Medical Sciences, 38(1), 22-29.

Selim, N. (2010). Cultural dimensions of depression in Bangladesh: A qualitative study in two villages of Matlab. Journal of Health Population and Nutrition, 28(1), 95-106.

Setiyawati, D., Blashki, G., Wraith, R., Colucci, E., \& Minas, H. (2014). Indonesian experts' perspectives on a curriculum for psychologists working in primary health care in Indonesia. Health Psychology \& Behavioural Medicine, 2(1), 623-639. https://doi.org/10.1080/21642850.2014.912946

Thornicroft, G., Mehta, N., Clement, S., Evans-Lacko, S., Doherty, M., Rose, D., \& Henderson, C. (2016). Evidence for effective interventions to reduce mental-healthrelated stigma and discrimination. Lancet, 387(10023), 1123-1132. https://doi.org/10.1016/S0140-6736(15)00298-6

Townsend, M., Kladder, V., Ayele, H., \& Mulligan, T. (2002). Systematic review of clinical trials examining the effects of religion on health. Southern Medical Journal, 95(12), 1429-1434.

Udedi, M. (2014). The prevalence of depression among patients and its detection by primary 
health care workers at Matawale Health Centre (Zomba). Malawi Medical Journal, 26(2), 34-37.

WHO. (2011). Mental Health ATLAS (Indonesia). Geneva: WHO.

Widiana, H. S., Manderson, L., \& Simpson, K. (2018). Experiences of depression in Yogyakarta, Indonesia. Mental Health, Religion \& Culture, 21(5), 470-483. https://doi.org/10.1080/13674676.2018.1486811

Widiana, H. S., Simpson, K., \& Manderson, L. (2018). Cultural expression of depression and the development of the Indonesian Depression Checklist. Transcultural Psychiatry, 55(3), 339-360. https://doi.org/10.1177/1363461518764491

Woodward, M. R. (1985). Healing and morality: A Javanese example. Social Science \& Medicine, 21(9), 1007-1021. https://doi.org/10.1016/0277-9536(85)90422-8

Worcester, S. (2013). New interview enhances DSM-5 cultural formulation. Family Practice News, 43(9), 16. 